\title{
Mitigating Error Propagation for Wireless Network Coding
}

\author{
Wei Guan, Student Member, IEEE, and K. J. Ray Liu, Fellow, IEEE
}

\begin{abstract}
Network coding has been widely used in wireless uplink to improve spectral efficiency. However, the relay decoding error may propagate to the intended receiver and thus severely degrade the diversity performance. So in this work, we develop two power scaling schemes at the relay side and two detection schemes at the receiver side, respectively, to mitigate error propagation and thus achieve full diversity for the twouser multiple access relay network. For the soft power scaling based link adaptive relaying, we develop a virtual source-relaydestination channel model and demonstrate that the relay power should be such to balance the signal-to-noise ratios of the sourcerelay channel and relay-destination channel. As for the hard power scaling based $\mathrm{ON}-\mathrm{OFF}$ relaying, we first design a decision rule based on total pairwise error probability, and then simplify it to the threshold-based relaying strategy. At the receiver side, we show that the weighted minimum distance detection with the weight being determined by the relative link quality of sourcerelay channel and relay-destination channel can achieve full diversity once the global channel state information is available, otherwise the maximum likelihood detection should be employed to achieve full diversity when the receiver only knows the local channel state information.
\end{abstract}

Index Terms-Network coding, multiple access, wireless relaying, diversity.

\section{INTRODUCTION}

$\mathbf{M}$ ULTIPLE antenna technology is a powerful tool to provide high transmission rate and reliable link connection by exploiting the rich spatial degree of freedom. However, equipping the user device with many co-located antennas is a technical challenge due to the high cost, high processing complexity and high power consumption. In a large network such as cellular system, the neighboring users can potentially behave as the distributed antennas for a source node by relaying signals to the destination, thus forming the so-called cooperative communications that can mitigate the needs of multiple physical antennas [1].

As it is generally hard for the user devices to transmit and receive on the same channel, half-duplex relaying protocols such as decode-and-forward (DF) and amplify-and-forward (AF) [2] have been widely studied in the community. Note that repetition coding is used in both $\mathrm{AF}$ and DF protocols, as the relay node either forwards an amplified version of the incoming signal or decode and re-encode the source message. As a result, each relay node has to use multiple orthogonal channels when forwarding messages for different sources, and

Manuscript received November 17, 2011; revised February 13 and April 10, 2012; accepted August 1, 2012. The associate editor coordinating the review of this paper and approving it for publication was D. Tuninetti.

The authors are with the Department of Electrical and Computer Engineering, University of Maryland, College Park, MD 20742 (e-mail: \{wguan, kjrliu\}@umd.edu).

Digital Object Identifier 10.1109/TWC.2012.083112.112053 the bandwidth efficiency is low. To overcome such shortcomings, network coding [3] has been actively extended to the wireless relaying systems. Although network coding was initially developed for the wired systems, it is equally suitable for the wireless applications. In fact, every node in a network can overhear multiple source messages due to the broadcast nature of the wireless medium. So instead of forwarding data to the individual destination separately, the relay node can mix these messages through superposition coding (e.g., bit-level XOR operation [3] or symbol-level linear combining [4]) so as to save the channel use.

Compared to its wired counterpart, wireless network coding suffers severe random decoding error due to channel fading. Indeed, it has been shown that forwarding the unreliable messages to the destination may lead to severe diversity loss [5][6]. Consequently, strong error detection/correction methods like cyclic redundancy check (CRC) should be exploited to prevent error propagation. For example, the authors in [7] demonstrate that when error detection at the destination is perfect, selectively decoding the signals on good links can achieve the same diversity order as the conventional selection relaying protocol while significantly improving the spectral efficiency. In [8], a protocol that opportunistically exploits the network coding gain is proposed, where the relay node forwards a single XORed packet based on the correctly decoded messages from multiple sources. For the multi-node cooperation scenario, [9] develops a set of diversity network codes that can maintain the diversity gain even when there is a limited number of failed links.

Although the CRC based selection relaying/combining methods are effective in controlling error propagation, they are not bandwidth efficient and would incur large decoding delay. Besides, as the whole packet would be dropped whenever failing the parity check, much power is wasted on decoding the unreliable packets, especially when the signal-to-noise ratio (SNR) is low. Consequently, mitigating error propagation through smart design of relaying and detection schemes is gaining more and more focus recently. For example, it is reported in [10]-[11] that threshold-based ON-OFF relaying can achieve full diversity order for DF relays. Likewise, [12] proposes another ON-OFF relaying protocol by exploiting the log-likelihood ratio metric for two-way relay channel. Instead of hard power scaling, [13] proposes a soft power scaling protocol based on the relative link quality of source-relay channel and relay-destination channel. At the receiver side, cooperative maximum-ratio combining [14][15] and maximum likelihood detector [16][17] are advocated to achieve full diversity.

We remark that most of the aforementioned methods (i.e., 
[10]-[11], [13]-[14] and [16]) are proposed for the repetition coding based DF relaying system where there is only a single source-destination pair. Although [12] talks about networkcoded system, the considered two-way relay channel is just a simple extension of DF relaying system that involves a single source-destination pair on each way. Consequently, these schemes are not directly applicable for a general multi-user network-coded system, which is potentially more vulnerable to relay decoding error. Perhaps the mostly related literatures are [15] and [17], which respectively extend the weighted combining technique [14] and maximum likelihood detector [16] to the network-coded uplink. However, perfect channel condition is assumed to be known in these literatures, and only receiver-side technique is considered, which largely limits their practical use. We also note that in many literatures focusing on network coding design (e.g., [8][9]), it is assumed that the decoding error can be perfectly detected/corrected, which is impractical in real systems. Indeed, when there does exist relay decoding error, the diversity performance of the network-coded system may severely degrade. So investigating efficient schemes to control error propagation in a multi-user network-coded system is an important issue that motivates the current work.

To be specific, we consider the wireless uplink channel where a single relay node helps the two source nodes send messages to a single destination by use of network coding. We first analyze the pairwise error probability (PEP) for binary phase-shift keying (BPSK) signal, and show that the dominant error event occurs when there is only one incorrectly decoded symbol at the destination. Then we proceed to develop some practical methods at the relay side and at the receiver side, respectively, to address the error propagation issue and thus achieve full diversity. At the relay side, we propose two power scaling schemes where the relay power is adaptive to the channel conditions. For the soft power scaling based link adaptive relaying (LAR), we first model the complicated source-relaydestination channel as a virtual point-to-point channel, and show that the relay power should be such to balance the SNRs of source-relay channel and relay-destination channel. As for the hard power scaling based ON-OFF relaying, we design a decision rule where the relay power is either full or zero depending on the total end-to-end PEP under each action, and we further derive a simplified threshold-based relaying scheme based on high-SNR approximation. At the receiver side, we show that the link adaptive combining (LAC) scheme that applies weighted minimum distance detection (MDD) with the weight being determined by the relative link quality of sourcerelay channel and relay-destination channel can achieve full diversity only when global channel state information (CSI) is available, otherwise maximum likelihood detection (MLD) should be employed to achieve full diversity when the receiver only knows local CSI. We also carefully analyze the relay power consumption and signalling overhead, and demonstrate how to extend our diversity analysis to the scenario using higher-order modulations.

The rest of this paper is organized as follows: We first describe the system model in Section II, and proceed to analyze the PEP in Section III. We then analyze the diversity performance of various schemes from Section IV to Section
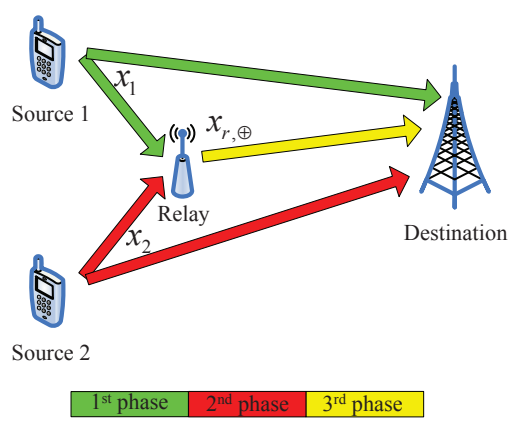

Fig. 1. System model for the wireless network-coded uplink.

VI. In Section VII, we shall present some simulation results, and some conclusions are given in Section VIII.

Notations: $|\cdot|$ and $(\cdot)^{*}$ stand for absolute value and conjugate, respectively. We shall use abbreviation i.i.d. for independent and identically distributed, and denote $Z \sim \mathcal{C N}\left(\mu, \sigma^{2}\right)$ as a circularly symmetric complex Gaussian random variable $Z$ with i.i.d. real part and imaginary part $\sim \mathcal{N}\left(\mu, \frac{\sigma^{2}}{2}\right)$. The probability of an event $\mathcal{A}$ and the probability density function (PDF) of a random variable $Z$ are denoted by $\operatorname{Pr}(\mathcal{A})$ and $f(Z)$, respectively. We define $Q(x)=\frac{1}{\sqrt{2 \pi}} \int_{x}^{\infty} e^{-\frac{t^{2}}{2}} d t$ as the Q-function, and denote $g\left(z, \sigma^{2}\right)=\frac{1}{\pi \sigma^{2}} \mathrm{e}^{-\frac{|z|^{2}}{\sigma^{2}}}$ as the PDF of $Z \sim \mathcal{C N}\left(0, \sigma^{2}\right)$. Finally, we say $h(x)=\mathrm{O}(g(x))$ if $\lim \sup _{x \rightarrow \infty} \frac{h(x)}{g(x)}<\infty$.

\section{SYSTEM MODEL}

Consider a wireless uplink channel with two source nodes sending data to a single destination. In the cellular systems, the two users usually access the base station through orthogonal channels to avoid interferences; however, the link is sometimes unreliable due to channel fading. The conventional relaying system can improve the link quality through spatial diversity. For half-duplex DF protocol, the relay node uses two orthogonal channels to receive and forward the messages for each source. A diversity order of 2 can be gained at a cost of doubled channel uses, as a total of 4 orthogonal channels is required. In this scenario, network coding can achieve the best tradeoff between spatial diversity and spectral efficiency, as the relay just sends a single XORed message that carries information of both source nodes, so it only needs 3 orthogonal channels as shown in Fig. 1. However, full diversity is not always achievable for this network-coded protocol due to random decoding error at the relay node. The basic issue we are striving to address in this work is how to achieve full diversity through smart design of relaying and detection schemes.

This 3-phase network-coded protocol works in the following way. In the $k$ th phase for $k=1,2$, the $k$ th source broadcasts its message to the relay and destination. The received signal can be represented as

$$
y_{k t}=h_{k t} \sqrt{P} x_{k}+n_{k t}=\bar{h}_{k t} x_{k}+n_{k t}
$$

for $t \in\{r, d\}$ and $k=1,2$. Here $y_{k t}$ is the received signal at node $t$ from source $k ; n_{k t} \sim \mathcal{C N}\left(0, N_{0}\right)$ is the additive noise; $h_{k t} \sim C N\left(0, \lambda_{k t}\right)$ is the rayleigh fading 
channel coefficient with $\lambda_{k t}$ being the channel gain; $P$ is the transmitted power; and $x_{k}$ is the source symbol with normalized power, i.e., $E\left|x_{k}\right|^{2}=1$. To facilitate the following analysis, we assume BPSK signal is used by the two sources, i.e., $x_{k} \in \Omega=\{1,-1\}$. The extension to higher-order modulations shall be discussed in later sections. For notational convenience, we also define the XORed source symbol as ${ }^{1}$ $x_{\oplus}=x_{1} \oplus x_{2}=-x_{1} x_{2}$. Note that $x_{\oplus} \in\{-1,1\}$ is also BPSK signal. Besides, we define $\bar{h}_{k t}=\sqrt{P} h_{k t}$ as the equivalent channel, and define $\gamma_{k t}=\left|h_{k t}\right|^{2} \Gamma$ as the instantaneous channel SNR with $\Gamma=\frac{P}{N_{0}}$ being the reference system SNR. It is easy to show that $\gamma_{k t}$ is an exponential random variable with mean $\Gamma_{k t}=\lambda_{k t} \Gamma$.

As the source symbols are randomly picked from the constellation with equal probability, MLD is equivalent to MDD at the relay node, i.e.,

$$
x_{r, k}=\arg \min _{\hat{x}_{k} \in \Omega}\left|y_{k r}-\bar{h}_{k r} \hat{x}_{k}\right|^{2}
$$

for $k=1,2$. Then, the decoded source messages are mixed through XOR operation, and the re-encoded message is $x_{r, \oplus}=$ $x_{r, 1} \oplus x_{r, 2}=-x_{r, 1} x_{r, 2}$. In the third phase, the relay node shall forward the network-coded message $x_{r, \oplus}$ to the destination, and the received signal is

$$
y_{r d}=h_{r d} \sqrt{\alpha P} x_{r, \oplus}+n_{r d}=\bar{h}_{r d} \sqrt{\alpha} x_{r, \oplus}+n_{r d} .
$$

Here $n_{r d} \sim \mathcal{C N}\left(0, N_{0}\right)$ is the additive noise, and $h_{r d} \sim$ $C N\left(0, \lambda_{r d}\right)$ is the rayleigh fading channel coefficient with $\lambda_{r d}$ being the channel gain. Besides, we define $\bar{h}_{r d}=\sqrt{P} h_{r d}$ as the equivalent relay-destination channel, and define $\gamma_{r d}=$ $\left|h_{r d}\right|^{2} \Gamma$ as the corresponding channel SNR that follows exponential distribution with mean $\Gamma_{r d}=\lambda_{r d} \Gamma$. Without loss of generality, we assume the additive noises and channel coefficients of different channels are all independent. Note that the power scaling coefficient $\alpha(0 \leq \alpha \leq 1)$ in (3) could be adaptive to channel conditions, as will be clear later.

As there is no error detection/correction code, neither the relay node nor the destination knows the decoding status of $x_{r, \oplus}$, i.e., whether $x_{r, \oplus}=x_{\oplus}$ or not. So weighted MDD can be employed at the destination to jointly decode the two source symbols based on the observations $y_{1 d}, y_{2 d}$ and $y_{r d}$, i.e.,

$$
\begin{aligned}
& \mathbf{x}_{d} \triangleq\left(x_{d, 1}, x_{d, 2}\right) \\
& =\arg \min _{\hat{x}_{1}, \hat{x}_{2} \in \Omega}\left(\sum_{k=1}^{2}\left|y_{k d}-\bar{h}_{k d} \hat{x}_{k}\right|^{2}+w\left|y_{r d}-\bar{h}_{r d} \sqrt{\alpha} \hat{x}_{\oplus}\right|^{2}\right),
\end{aligned}
$$

where the combining weight $w$ can be leveraged to account for the possible relay decoding error, as will be clear later. Our major contribution of this work is to design some special power scaling coefficient $\alpha$ and combining weight $w$ such that the whole system can achieve full diversity.

We remark that the link adaptive techniques we shall discuss later depends largely on how much CSI is known at each node. For the local CSI based methods, we assume that the receiver of each channel knows the corresponding instantaneous

\footnotetext{
${ }^{1}$ Note that the conventional XOR operation is performed at bit-level. Here we omit the bit-to-symbol mapping and use the equivalent symbol-level XORed output directly.
}

channel coefficient (or equivalently, the instantaneous channel SNR). Specifically, $\bar{h}_{k r}\left(\bar{\gamma}_{k r}\right)$ are known at the relay node for $k=1,2$, and/or $\bar{h}_{k d}\left(\gamma_{k d}\right)$ and $\bar{h}_{r d}\left(\gamma_{r d}\right)$ are known at the destination for $k=1,2$. For the global CSI based methods, we further assume that the relay node knows $\bar{h}_{r d}\left(\gamma_{r d}\right)$ and/or the destination knows $\bar{h}_{k r}\left(\gamma_{k r}\right)$ for $k=1,2$ besides the local CSI. As the average channel SNRs are second-order statistics that stay stationary over a long time, we assume that they are available to all nodes with trivial feedback overhead.

\section{ERROR PERFORMANCE ANALYSIS}

Before proceeding to discuss the detailed design of relaying and detection schemes, we first analyze in this section the endto-end error performance. In this work, we are interested in the diversity order of the error performance, which is defined as

$$
d i v=-\log _{\Gamma \rightarrow \infty} \frac{\log \operatorname{Pr}\left(\mathbf{x}_{\mathbf{d}} \neq \mathbf{x}\right)}{\log \Gamma}
$$

where $\mathbf{x}=\left(x_{1}, x_{2}\right)$ is the source symbol vector. Note that the maximum diversity order is 2 because each source symbol can reach the receiver through two independent channels, i.e., the individual direct link and the common relay branch, as the network-coded symbol provides side information for both sources. Unfortunately, the exact error analysis is intractable due to the complexity in deriving the closed-form decision regions of (4). Alternatively, we shall investigate the pairwise error probability (PEP), which is well known to be a tight bound on the real error probability.

Using the law of total probability, we can express the PEP as

$$
\begin{aligned}
\operatorname{Pr}(\mathbf{x} & \rightarrow \hat{\mathbf{x}})=\operatorname{Pr}\left(\mathbf{x} \rightarrow \hat{\mathbf{x}}, \Phi_{\text {prop }}, \Phi_{\text {on }}\right) \\
& +\operatorname{Pr}\left(\mathbf{x} \rightarrow \hat{\mathbf{x}}, \Phi_{\text {free }}, \Phi_{\text {on }}\right)+\operatorname{Pr}\left(\mathbf{x} \rightarrow \hat{\mathbf{x}}, \Phi_{o f f}\right)
\end{aligned}
$$

Here $\Phi_{o n}$ and $\Phi_{\text {off }}$ is the event that the relay node does forward the message (i.e., $\alpha \neq 0$ ) and stays idle (i.e., $\alpha=$ 0 ), respectively. In the case of $\alpha=0$, the weight $w$ in (4) should be set to 0 too as there is no information sent from the relay node at all. On the other hand, $\Phi_{\text {free }}$ is the event that the relay node obtains the correct network-coded symbol (i.e., $x_{r, \oplus}=x_{\oplus}$ ), and $\Phi_{\text {prop }}$ means $x_{r, \oplus} \neq x_{\oplus}$ and the relay decoding error may propagate to the destination. According to the definition, we have

$$
\begin{aligned}
\operatorname{Pr}\left(\Phi_{\text {prop }}\right)= & \operatorname{Pr}\left(x_{r, 1}=x_{1}\right) \operatorname{Pr}\left(x_{r, 2} \neq x_{2}\right) \\
& +\operatorname{Pr}\left(x_{r, 1} \neq x_{1}\right) \operatorname{Pr}\left(x_{r, 2}=x_{2}\right) \\
= & \frac{1}{2}\left(1-\sqrt{\frac{\Gamma_{1 r}}{1+\Gamma_{1 r}}} \sqrt{\frac{\Gamma_{2 r}}{1+\Gamma_{2 r}}}\right) \\
\Gamma & \stackrel{\approx}{\approx} \frac{\lambda_{1 r}+\lambda_{2 r}}{4 \lambda_{1 r} \lambda_{2 r}} \Gamma^{-1},
\end{aligned}
$$

and $\operatorname{Pr}\left(\Phi_{\text {free }}\right)=1-\operatorname{Pr}\left(\Phi_{\text {prop }}\right)$.

After some manipulations, it is also straightforward to 
obtain the conditional PEPs as ${ }^{2}$

$$
\left\{\begin{array}{r}
\operatorname{Pr}(\mathbf{x} \rightarrow-\mathbf{x} \mid h)=Q\left(\sqrt{2 \sum_{k \in\{1,2\}} \gamma_{k d}}\right), \\
\operatorname{Pr}\left(\mathbf{x} \rightarrow\left(-x_{1}, x_{2}\right) \mid \Phi_{o f f}, h\right)=Q\left(\sqrt{2 \gamma_{1 d}}\right), \\
\operatorname{Pr}\left(\mathbf{x} \rightarrow\left(-x_{1}, x_{2}\right) \mid \Phi_{\text {free }}, \Phi_{o n}, h\right) \\
=Q\left(\frac{\sqrt{2}\left(\gamma_{1 d}+\alpha w \gamma_{r d}\right)}{\sqrt{\gamma_{1 d}+\alpha w^{2} \gamma_{r d}}}\right), \\
\operatorname{Pr}\left(\mathbf{x} \rightarrow\left(-x_{1}, x_{2}\right) \mid \Phi_{\text {prop }}, \Phi_{o n}, h\right) \\
=Q\left(\frac{\sqrt{2}\left(\gamma_{1 d}-\alpha w \gamma_{r d}\right)}{\sqrt{\gamma_{1 d}+\alpha w^{2} \gamma_{r d}}}\right) .
\end{array}\right.
$$

By using the integral representation of Q-function [18]

$$
Q(x)=\frac{1}{\pi} \int_{0}^{\pi / 2} \exp \left(-\frac{x^{2}}{2 \sin ^{2} \theta}\right) d \theta
$$

and averaging (8a) and (8b) over channel distributions, we can further obtain

$$
\left\{\begin{array}{l}
\operatorname{Pr}(\mathbf{x} \rightarrow-\mathbf{x}) \stackrel{\Gamma \rightarrow \infty}{\approx} \frac{3}{16 \lambda_{1 d} \lambda_{2 d}} \Gamma^{-2}, \\
\operatorname{Pr}\left(\mathbf{x} \rightarrow\left(-x_{1}, x_{2}\right) \mid \Phi_{\text {off }}\right) \stackrel{\Gamma \vec{\approx}^{\infty}}{\approx} \frac{1}{4 \lambda_{1 d}} \Gamma^{-1} .
\end{array}\right.
$$

It is observed that the error event that both of the two source symbols are decoded incorrectly at the receiver has the same conditional probability under any relaying status as shown in (8a), and the corresponding diversity order is always 2 . Therefore, the dominant error event occurs when only one of the source symbols flips at the receiver, which determines the overall diversity performance. As the power scaling coefficient $\alpha$ and the combining weight $w$ may depend on channel conditions too, we shall derive the unconditional PEPs of (8c) and (8d) in later sections when discussing the detailed scheme design.

\section{RELAY-SIDE SCHEMES}

In this section, we shall develop two power scaling schemes at the relay side. For both methods, the combining weight $w$ in (4) is set to 1, i.e., the regular MDD with equal weights is employed at the receiver. We demonstrate that full diversity can be achieved by smartly designing the power scaling coefficient $\alpha$ according to channel conditions.

\section{A. Link Adaptive Relaying (LAR)}

LAR was first proposed in [13] for the single-source DF system. The idea is to adapt the relay power to the channel conditions so as to limit the interference of relay decoding error. However, LAR cannot be employed directly in the network-coded system, which is usually associated with multiple source-relay channels.

To extend the spirit of LAR, we first develop a virtual channel model for the source-relay-destination link, as shown in Fig. 2. For the real link in Fig. 2(a), the relay node simply forwards an estimate $x_{r, \oplus}$ of $x_{\oplus}$ to the destination, which

\footnotetext{
${ }^{2}$ The symbol $h$ means the probability is conditional on the related channels. Same convention is used throughout this work.
}

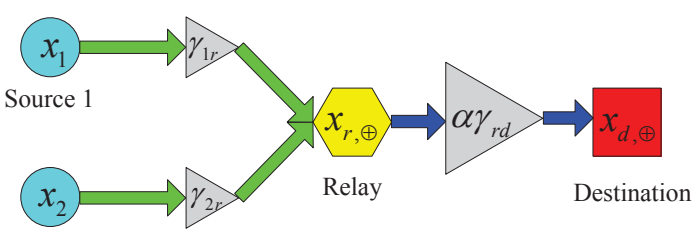

Source 2

(a) Real source-relay-destination channel

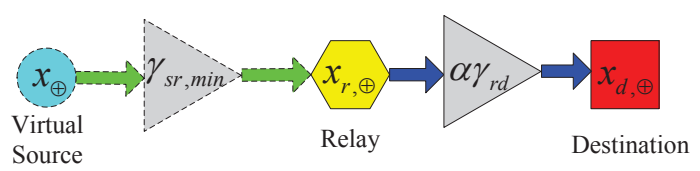

(b) Virtual source-relay channel

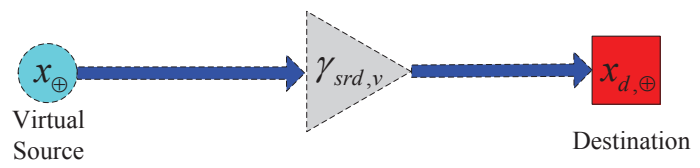

(c) Virtual source-relay-destination channel

Fig. 2. Virtual channel model.

is not totally reliable but still provides side information of source symbols. Suppose now the destination just decodes $x_{\oplus}$ as $x_{d, \oplus}$ based on the observation $y_{r, d}$, then the end-to-end BER $\operatorname{Pr}\left(x_{d, \oplus} \neq x_{\oplus}\right)$ is a good measure of the reliability of this two-hop relay branch. To this end, we first approximate the conditional relay decoding error probability as

$$
\begin{aligned}
& \operatorname{Pr}\left(x_{r, \oplus} \neq x_{\oplus} \mid h\right) \\
& =\operatorname{Pr}\left(x_{r, 1}=x_{1} \mid h\right) \operatorname{Pr}\left(x_{r, 2} \neq x_{2} \mid h\right) \\
& \quad+\operatorname{Pr}\left(x_{r, 1} \neq x_{1} \mid h\right) \operatorname{Pr}\left(x_{r, 2}=x_{2} \mid h\right) \\
& =Q\left(\sqrt{2 \gamma_{1 r}}\right)+Q\left(\sqrt{2 \gamma_{2 r}}\right)-2 Q\left(\sqrt{2 \gamma_{1 r}}\right) Q\left(\sqrt{2 \gamma_{2 r}}\right) \\
& \approx Q\left(\sqrt{2 \gamma_{s r, \text { min }}}\right),
\end{aligned}
$$

where $\gamma_{s r, \text { min }}=\min \left(\gamma_{1 r}, \gamma_{2 r}\right)$ represents the SNR of the worse source-relay channel. As $\gamma_{1 r}$ and $\gamma_{2 r}$ are independent exponential random variables, $\gamma_{s r, \min }$ is also an exponential random variable with mean $\Gamma_{s r, \text { min }}=\lambda_{s r, \min } \Gamma$, where $\lambda_{s r, \min }=\frac{\lambda_{1 r} \lambda_{2 r}}{\lambda_{1 r}+\lambda_{2 r}}$. Such approximation is quite tight when both of $\gamma_{1 r}$ and $\gamma_{2 r}$ and their difference are reasonably large, as the Q-function $Q(x)$ decays really fast with $x$. The above approximation shows that the multiple-input singleoutput source-relay channel can be accurately characterized by a single-input single-output virtual channel with the channel input being the XORed source message $x_{\oplus}$ and the channel SNR being $\gamma_{s r, m i n}$, as shown in Fig. 2(b). This virtual channel model can be justified by observing that the conditional BER $\operatorname{Pr}\left(x_{r, \oplus} \neq x_{\oplus} \mid h\right)$ over the virtual source-relay channel, which happens to be $Q\left(\sqrt{2 \gamma_{s r, m i n}}\right)$, is approximately the same as that over the real one. In a similar way, the end-toend BER of this two-hop relay branch can be approximated as

$$
\begin{aligned}
& \operatorname{Pr}\left(x_{d, \oplus} \neq x_{\oplus} \mid h\right) \\
& =\operatorname{Pr}\left(x_{d, \oplus} \neq x_{r, \oplus} \mid h\right) \operatorname{Pr}\left(x_{r, \oplus}=x_{\oplus} \mid h\right) \\
& \quad+\operatorname{Pr}\left(x_{d, \oplus}=x_{r, \oplus} \mid h\right) \operatorname{Pr}\left(x_{r, \oplus} \neq x_{\oplus} \mid h\right) \\
& \approx Q\left(\sqrt{2 \gamma_{s r d, v}}\right),
\end{aligned}
$$




$$
\operatorname{Pr}\left(\Phi_{\text {prop }} \mid h_{1 r}, h_{2 r}\right) \leq \frac{\sum_{\hat{\mathbf{x}} \neq \mathbf{x}}\left(\operatorname{Pr}\left(\mathbf{x} \rightarrow \hat{\mathbf{x}} \mid \Phi_{\text {off }}\right)-\operatorname{Pr}\left(\mathbf{x} \rightarrow \hat{\mathbf{x}} \mid \Phi_{\text {free }}, \Phi_{\text {on }}\right)\right)}{\sum_{\hat{\mathbf{x}} \neq \mathbf{x}}\left(\operatorname{Pr}\left(\mathbf{x} \rightarrow \hat{\mathbf{x}} \mid \Phi_{\text {prop }}, \Phi_{o n}\right)-\operatorname{Pr}\left(\mathbf{x} \rightarrow \hat{\mathbf{x}} \mid \Phi_{\text {free }}, \Phi_{\text {on }}\right)\right)},
$$

where $\gamma_{s r d, v}=\min \left(\gamma_{s r, \min }, \alpha \gamma_{r d}\right)$. Using the same arguments, we can further model this two-hop branch as a pointto-point virtual link with the channel input being $x_{\oplus}$ and the equivalent channel SNR being $\gamma_{s r d, v}$, as shown in Fig. 2(c). Note that the link quality is uniquely characterized by this virtual SNR, which is independent of the relay decoding error patterns. Clearly, when $\gamma_{s r, \text { min }} \leq \gamma_{r d}$, the sourcerelay channel becomes the bottleneck, so increasing $\alpha$ beyond $\frac{\gamma_{s r, \min }}{\gamma_{r d}}$ makes no sense as $\gamma_{s r d, v} \equiv \gamma_{s r, \min }$. On the other hand, if $\gamma_{s r, \min } \geq \gamma_{r d}$, then the relay-destination channel becomes the bottleneck and the relay node should forward the message with full power. With the above observation, we can design the power scaling coefficient $\alpha$ as

$$
\alpha=\left\{\begin{array}{l}
\min \left(\frac{\gamma_{s r, m i n}}{\gamma_{r d}}, 1\right), \text { global CSI } \\
\min \left(\frac{\gamma_{s r, m i n}}{\Gamma_{r d}}, 1\right), \text { local CSI }
\end{array}\right.
$$

Note that $\gamma_{r d}$ is unknown to the relay node when only local CSI is available, so we have used its mean $\Gamma_{r d}$ in (13b) as a blind estimate. In some sense, the relay node behaves like a link coordinator that strives to balance the channel SNRs of the two hops, as the worse hop limits the whole link quality. As for the diversity performances, we have the following proposition.

Proposition 1: Both of the global CSI based LAR and local CSI based LAR can achieve a diversity order of 2 .

$$
\text { Proof: See Appendix A. }
$$

\section{B. ON-OFF Relaying}

Different from soft power scaling in LAR, the relay node could instead apply hard power scaling (i.e., $\alpha \in\{0,1\}$ ). As the total PEP upper bounds the real decoding error probability at the receiver, we propose to turn on the relay node (i.e., $\alpha=1$ ) when

$$
\sum_{\hat{\mathbf{x}} \neq \mathbf{x}} \operatorname{Pr}\left(\mathbf{x} \rightarrow \hat{\mathbf{x}} \mid \Phi_{o n}, h_{1 r}, h_{2 r}\right) \leq \sum_{\hat{\mathbf{x}} \neq \mathbf{x}} \operatorname{Pr}\left(\mathbf{x} \rightarrow \hat{\mathbf{x}} \mid \Phi_{o f f}\right) .
$$

That is, the relay node always chooses the action that promises smaller total PEP. If (14) is otherwise false, then the relay node should stay idle by letting $\alpha=0$. After some manipulations, we can show that the above decision rule is equivalent to (15) shown on the top of this page, where $\operatorname{Pr}\left(\Phi_{\text {prop }} \mid h_{1 r}, h_{2 r}\right)$ is given by (11); $\operatorname{Pr}(\mathbf{x} \rightarrow-\mathbf{x})$ and $\operatorname{Pr}\left(\mathbf{x} \rightarrow\left(-x_{1}, x_{2}\right) \mid \Phi_{\text {off }}\right)$ is shown in (10a) and (10b), respectively; and after plugging $\alpha=w=1$ back into (8c) and (8d) and averaging over channel distribution, we have

$$
\left\{\begin{array}{l}
\operatorname{Pr}\left(\mathbf{x} \rightarrow\left(-x_{1}, x_{2}\right) \mid \Phi_{\text {free }}, \Phi_{\text {on }}\right) \stackrel{\Gamma \rightarrow \infty}{\underset{\approx}{*}} \frac{3 \Gamma^{-2}}{16 \lambda_{1 d} \lambda_{r d}},(16 \mathrm{a}) \\
\operatorname{Pr}\left(\mathbf{x} \rightarrow\left(-x_{1}, x_{2}\right) \mid \Phi_{\text {prop }}, \Phi_{\text {on }}\right) \stackrel{\Gamma \rightarrow \infty}{\overbrace{}^{\infty}} \frac{\lambda_{r d}}{\lambda_{1 d}+\lambda_{r d}},(16 \mathrm{~b})
\end{array}\right.
$$

where the high-SNR approximation in (16b) has been proved in [10]. Note that the exact decision rule (15) is somewhat intractable, as the average of Q-function over channel distribution is hard to manipulate. Alternatively, we choose to use the high-SNR approximations to simplify the right-hand side of (15), i.e.,

$$
\left\{\begin{array}{l}
\sum_{\hat{\mathbf{x}} \neq \mathbf{x}} \operatorname{Pr}\left(\mathbf{x} \rightarrow \hat{\mathbf{x}} \mid \Phi_{o f f}\right) \stackrel{\Gamma \rightarrow \infty}{\approx} \frac{\lambda_{1 d}+\lambda_{2 d}}{4 \lambda_{1 d} \lambda_{2 d}} \Gamma^{-1} \\
\sum_{\hat{\mathbf{x}} \neq \mathbf{x}} \operatorname{Pr}\left(\mathbf{x} \rightarrow \hat{\mathbf{x}} \mid \Phi_{\text {prop }}, \Phi_{o n}\right) \stackrel{\Gamma \rightarrow \infty}{\approx} \sum_{k=1}^{2} \frac{\lambda_{r d}}{\lambda_{k d}+\lambda_{r d}} \\
\sum_{\hat{\mathbf{x}} \neq \mathbf{x}} \operatorname{Pr}\left(\mathbf{x} \rightarrow \hat{\mathbf{x}} \mid \Phi_{\text {free }}, \Phi_{o n}\right) \\
\stackrel{\Gamma \rightarrow \infty}{\approx} \frac{\lambda_{1 d}+\lambda_{2 d}+\lambda_{r d}}{\lambda_{1 d} \lambda_{2 d} \lambda_{r d}} \frac{3}{16} \Gamma^{-2}
\end{array}\right.
$$

By using the virtual source-relay channel model in Fig. 2(b) and applying the Chernoff bound, we can further simplify the decision rule (15) as

$$
Q\left(\sqrt{2 \gamma_{s r, \text { min }}}\right) \leq \frac{1}{2} e^{-\gamma_{s r, \text { min }}} \leq \frac{1}{2 \lambda_{T}} \Gamma^{-1},
$$

or equivalently,

$$
\gamma_{s r, \min } \geq \log \lambda_{T} \Gamma
$$

where

$$
\lambda_{T}=\frac{2 \lambda_{1 d} \lambda_{2 d} \lambda_{r d}\left(\lambda_{1 d}+\lambda_{2 d}+2 \lambda_{r d}\right)}{\left(\lambda_{1 d}+\lambda_{2 d}\right)\left(\lambda_{1 d}+\lambda_{r d}\right)\left(\lambda_{2 d}+\lambda_{r d}\right)}
$$

is a constant determined by the second-order statistics. Consequently, the complex decision rule (14) is simplified to the threshold-based relaying strategy. We observe that the two source-relay channels have to meet the same SNR threshold, as the relay decoding error is bounded by the worse channel as shown in (11). We also observe that imposing any threshold having the form of $\log \left(\lambda_{T, k} \Gamma\right)$ on $\gamma_{k}$ for $\lambda_{T, k}>0$ and $k=1,2$ would lead to the same diversity performance, since $\log \lambda_{T, k} \Gamma \stackrel{\Gamma \underset{\sim}{\approx}}{\approx} \log \Gamma$. The special $\lambda_{T}$ given in (20) can be justified by the following proposition.

Proposition 2: For all the ON-OFF relaying protocols with $\Phi_{o n}=\left\{\gamma_{k r} \geq \log \lambda_{T, k} \Gamma, k=1,2\right\}$, where $\lambda_{T, k}$ is any positive constant, a diversity order of 2 can be achieved. Besides, $\lambda_{T, 1}=\lambda_{T, 2}=\lambda_{T}$ is optimum in the sense of minimizing the total end-to-end PEP.

Proof: See Appendix B.

\section{RECEIVER-Side SCHEMES}

So far we have shown that the error propagation issue could be efficiently addressed at the relay side. Alternatively, we shall show in this section that full diversity can also be achieved through receiver-side processing even when there is no power scaling at the relay side. Throughout this section, we assume the relay node always forwards message using full power (i.e., $\alpha=1$ ). 


\section{A. Link Adaptive Combining (LAC)}

In LAR and ON-OFF relaying, the receiver implicitly assume there is no relay decoding error, thus the combining weight $w$ is always set to 1 . Basically, the combining weight is a kind of confidence measure that reflects how reliable the relay branch is. When the relay decoding error occurs with high probability, the destination should adaptively lower the combining weight to discount the contribution of the relay branch.

Before describing our choice of $w$, let us first revisit the virtual relay branch shown in Fig. 2(c) to gain more insights. As mentioned before, this virtual channel has the BPSK input $x_{\oplus}$ and the channel SNR is $\gamma_{s r d, v}$. As the relaydestination channel coefficient is $h_{r d}$, we can approximate the real received signal $y_{r d}$ in (3) as

$$
\tilde{y}_{r d}=\bar{h}_{r d} \sqrt{\alpha} x_{\oplus}+\tilde{n}_{r d}
$$

where $\tilde{n}_{r d} \sim \mathcal{C N}\left(0, \frac{\left|\bar{h}_{r d}\right|^{2} \alpha}{\gamma_{s r d, v}}\right)$ is the virtual channel noise, and the noise power is such that the SNR of this virtual signal is exactly $\gamma_{s r d, v}$. With the above signal model, it is easy to show that the MLD based on the observations $y_{1 d}, y_{2 d}$ and $\tilde{y}_{r d}$ is

$$
\begin{array}{r}
\mathbf{x}_{d} \triangleq\left(x_{d, 1}, x_{d, 2}\right)=\arg \max _{\hat{x}_{1}, \hat{x}_{2} \in \Omega} f\left(y_{1 d}, y_{2 d}, \tilde{y}_{r d} \mid \hat{x}_{1}, \hat{x}_{2}\right) \\
=\arg \max _{\hat{x}_{1}, \hat{x}_{2} \in \Omega} g\left(\tilde{y}_{r d}-\bar{h}_{r d} \sqrt{\alpha} \hat{x}_{\oplus}, \frac{\left|\bar{h}_{r d}\right|^{2} \alpha}{\gamma_{s r d, v}}\right) \\
\times \prod_{k \in\{1,2\}} g\left(y_{k d}-\bar{h}_{k d} \hat{x}_{k}, N_{0}\right),
\end{array}
$$

where we exploit the independence of the three received signals. As $\alpha=1$, we can show that the above MLD is actually equivalent to the weighted MDD in (4) by letting

$$
w=\frac{\gamma_{s r d, v}}{\gamma_{r d}}=\min \left(\frac{\gamma_{s r, \min }}{\gamma_{r d}}, 1\right) .
$$

We remark that our design is asymptotically the same as that proposed in [15]; however, unlike [15] which directly extends the scheme in [14] in a heuristic way, we justify such design using our virtual channel model, which clearly shows that the adaptive weight should be such to equalize the virtual channel noise power before entering the combiner. To be specific, when $\gamma_{s r, \min } \leq \gamma_{r d}$, the virtual noise power is $\frac{\gamma_{r d}}{\gamma_{s r, m i n}} N_{0} \geq N_{0}$, which reflects the fact that the delivered symbol $x_{\oplus}$ is unreliable as the source-relay channel is the system bottleneck. On the other hand if $\gamma_{s r, \text { min }}>\gamma_{r d}$, the relay-destination channel becomes the bottleneck, then the virtual noise has power $N_{0}$ and the relay branch is given full credit as the other two source-destination channels.

By comparing global CSI based LAR (13a) and global CSI based LAC scheme (23), we observe that

$$
\alpha w \gamma_{r d}=\gamma_{s r d, v}=\min \left(\gamma_{s r, m i n}, \gamma_{r d}\right)
$$

in both schemes. This factor can be regarded as the aggregate scaling coefficient effective on $x_{r, \oplus}$ to mitigate the impact of relay decoding error. So basically, the two schemes are following the same principle to address the error propagation issue, and this design goal can be fulfilled either at the relay side (i.e., LAR) or at the receiver side (i.e., LAC). Due to such relations, one may guess that when only local CSI is available, we can replace $\gamma_{s r, \min }$ by its average $\Gamma_{s r, \min }$ in (23), i.e., let

$$
w=\min \left(\frac{\Gamma_{s r, \min }}{\gamma_{r d}}, 1\right)
$$

and still achieve a diversity order of 2 as is the case of local CSI based LAR scheme. However, this is not true as we show in the following proposition.

Proposition 3: For global CSI based LAC scheme (23), a diversity order of 2 can be achieved; however, the diversity order of local CSI based LAC scheme (25) is only 1.

Proof: The first part is easy to prove, as the PEP upper bound is the same as (36) after plugging (24) into (35). So let us focus on the local CSI based LAC. As $\alpha=1$ and $\operatorname{Pr}\left(\Phi_{o f f}\right)=0$, we derive from (6)

$$
\begin{aligned}
& \operatorname{Pr}\left(\mathbf{x} \rightarrow\left(-x_{1}, x_{2}\right)\right) \\
& \quad \geq \operatorname{Pr}\left(\mathbf{x} \rightarrow\left(-x_{1}, x_{2}\right) \mid \Phi_{\text {prop }}, \Phi_{\text {on }}\right) \operatorname{Pr}\left(\Phi_{\text {prop }}\right) .
\end{aligned}
$$

After plugging $\alpha=1$ and (25) back into (8d), we have

$\operatorname{Pr}\left(\mathbf{x} \rightarrow\left(-x_{1}, x_{2}\right) \mid \Phi_{\text {prop }}, \Phi_{\text {on }}\right)$

$=E\left[Q\left(\frac{\sqrt{2}\left(\gamma_{1 d}-\min \left(\Gamma_{s r, m i n}, \gamma_{r d}\right)\right)}{\sqrt{\gamma_{1 d}+\min \left(\Gamma_{s r, m i n}^{2}, \gamma_{r d}^{2}\right) / \gamma_{r d}}}\right)\right]$

$\geq E_{\gamma_{1 d}<\Gamma_{s r, \min } \leq \gamma_{r d}}\left[Q\left(\frac{\sqrt{2}\left(\gamma_{1 d}-\min \left(\Gamma_{s r, \min }, \gamma_{r d}\right)\right)}{\sqrt{\gamma_{1 d}+\min \left(\Gamma_{s r, \min }^{2}, \gamma_{r d}^{2}\right) / \gamma_{r d}}}\right)\right]$

$\geq \frac{1}{2} \operatorname{Pr}\left(\gamma_{r d}>\Gamma_{s r, \min }, \gamma_{1 d}<\Gamma_{s r, \min }\right)$

$=\frac{1}{2} e^{-\frac{\lambda_{s r, m i n}}{\lambda_{r d}}}\left(1-e^{-\frac{\lambda_{s r, m i n}}{\lambda_{1 d}}}\right)=\mathrm{O}(1)$,

where in the last inequality we use the fact $Q(x) \geq \frac{1}{2}$ for $x \leq 0$. Recall that $\operatorname{Pr}\left(\Phi_{\text {prop }}\right)=\mathrm{O}\left(\Gamma^{-1}\right)$ as shown in (7), we conclude that $\operatorname{Pr}\left(\mathbf{x} \rightarrow\left(-x_{1}, x_{2}\right)\right)=\mathrm{O}\left(\Gamma^{-1}\right)$.

\section{B. Maximum Likelihood Detection (MLD)}

So far, we have assumed that the linear combiner is employed at the destination; however, full diversity cannot be achieved when the receiver only knows local CSI. So in this subsection, we study the diversity performances of MLD, which is optimum in the sense of minimizing detection errors.

The MLD based on real observations $y_{1 d}, y_{2 d}$ and $y_{r d}$ can be expressed as

$$
\begin{aligned}
\mathbf{x}_{d} & \triangleq\left(x_{d, 1}, x_{d, 2}\right) \\
& =\arg \max _{\hat{x}_{1}, \hat{x}_{2} \in \Omega} f\left(y_{r d} \mid \hat{x}_{1}, \hat{x}_{2}\right) \prod_{k \in\{1,2\}} g\left(y_{k d}-\bar{h}_{k d} \hat{x}_{k}, N_{0}\right),
\end{aligned}
$$

where

$$
\begin{aligned}
f\left(y_{r d} \mid x_{1}, x_{2}\right)= & g\left(y_{r d}+\bar{h}_{r d} x_{\oplus}, N_{0}\right) \operatorname{Pr}\left(\Phi_{\text {prop }}\right) \\
& +g\left(y_{r d}-\bar{h}_{r d} x_{\oplus}, N_{0}\right) \operatorname{Pr}\left(\Phi_{\text {free }}\right)
\end{aligned}
$$

is the conditional PDF of $y_{r d}$ given the two source symbols $x_{1}$ and $x_{2}$, and (11) and (7) should be plugged into the term $\operatorname{Pr}\left(\Phi_{\text {prop }}\right)$ for global CSI based MLD and local CSI based MLD, respectively. Our main result is summarized below. 


$$
\begin{aligned}
\operatorname{Pr}\left(x_{r, k} \neq x_{k} \mid \Phi_{o n}\right) & =\frac{1}{\pi} \int_{0}^{\frac{M-1}{M} \pi} \int_{g_{p s k}^{-1} \log \Gamma}^{\infty} \exp \left(-\frac{g_{p s k} \gamma}{\sin ^{2} \theta}\right) \frac{1}{\lambda_{k r} \Gamma} \exp \left(-\frac{\gamma-g_{p s k}^{-1} \log \Gamma}{\lambda_{k r} \Gamma}\right) d \gamma d \theta \\
& =\frac{1}{\pi} \int_{0}^{\frac{M-1}{M} \pi} \frac{\sin ^{2} \theta}{g_{p s k} \lambda_{k r} \Gamma+\sin ^{2} \theta} \exp \left(-\frac{\log \Gamma}{\sin ^{2} \theta}\right) d \theta \leq \frac{1}{\pi g_{p s k} \lambda_{k r} \Gamma} \int_{0}^{\frac{M-1}{M} \pi} \exp \left(-\frac{\log \Gamma}{\sin ^{2} \theta}\right) d \theta \\
& \leq \frac{1}{\pi g_{p s k} \lambda_{k r} \Gamma} \exp (-\log \Gamma) \frac{M-1}{M} \pi=\frac{M-1}{M g_{p s k} \lambda_{k r}} \Gamma^{-2} .
\end{aligned}
$$

Proposition 4: Both of the global CSI based MLD and local CSI based MLD can achieve a diversity order of 2 . Proof: See Appendix C.

\section{More Discussions}

In this section, we shall compare the aforementioned schemes in terms of relay power consumption and signalling overhead. We also briefly discuss the extension to higher-order modulations.

\section{A. Relay Power Consumption Ratio}

For the relay-side schemes, the relay power is adaptively scaled by the coefficient $\alpha$. To compare the relay power consumption of different schemes, we define the relay power consumption ratio as $\bar{\alpha}=E(\alpha)$. Under this definition, the relay power consumption ratio of the receiver-side schemes is 1 as the relay node always sends message with full power (i.e., $\alpha=1$ ). After some manipulations, we can show that

$$
\left\{\begin{aligned}
\bar{\alpha}_{O N-O F F} & \approx \operatorname{Pr}\left(\gamma_{s r, m i n} \geq \log \lambda_{T} \Gamma\right) \\
& =\exp \left(-\frac{\log \lambda_{T} \Gamma}{\lambda_{s r, m i n} \Gamma}\right) \stackrel{\Gamma \rightarrow \infty}{\approx} 1, \\
\bar{\alpha}_{L A R, \text { Local }} & =\frac{\lambda_{s r, m i n}}{\lambda_{r d}}\left(1-e^{-\frac{\lambda_{r d}}{\lambda_{s r, m i n}}}\right), \\
\bar{\alpha}_{L A R, \text { Global }} & =\frac{\lambda_{s r, \text { min }}}{\lambda_{r d}} \log \left(\frac{\lambda_{r d}}{\lambda_{s r, m i n}}+1\right) .
\end{aligned}\right.
$$

Clearly, for the hard power scaling based ON-OFF relaying, the relay power consumption increases as the relay decoding error probability reduces with SNR. On the contrary, the relay power consumption ratio is independent of SNR for the soft power scaling based LAR, as it is adaptive to the relative quality of source-relay channel and relay-destination channel. When the source-relay channel is much better than the relaydestination channel, we have $\frac{\lambda_{s r, m i n}}{\lambda_{r d}} \rightarrow \infty$ and $\bar{\alpha}_{L A R} \rightarrow 1$. On the contrary, if the source-relay channel is the bottleneck, we have $\frac{\lambda_{s r, \text { min }}}{\lambda_{r d}} \rightarrow 0$ and $\bar{\alpha}_{L A R} \rightarrow 0$, in which case the network-coded uplink is reduced to the conventional timedivision multiple access without node cooperation.

\section{B. Signalling Overhead}

The CSI assumptions directly determine the signalling overhead of the whole system. For the ON-OFF relaying, as local CSI is exploited and $\alpha \in\{0,1\}$, the relay node only needs to send 1 bit indicating ON or OFF to the destination. As for the global CSI based LAR, the destination has to feed $\gamma_{r d}$ back to the relay node, which then sends back the calculated power scaling coefficient $\alpha$. So the signalling overhead depends largely on the quantization accuracy of $\alpha$ and $\gamma_{r d}$. The story is totally different for the local CSI based LAR. Indeed, after the relay node estimates the source-relay channel coefficients, it can compute the power scaling coefficient $\alpha$ and effect it on the training sequence sent to the destination. After that, the destination can obtain the equivalent channel coefficient $\bar{h}_{r d} \sqrt{\alpha}$ that is needed for MDD. Consequently, there is no additional signalling overhead for the local CSI based LAR, which is also the case for the local CSI based MLD as the destination only exploits the average source-relay channel gain. Finally for the global CSI based LAC and global CSI based MLD, the relay node needs to report the source-relay channel SNR to the destination.

\section{Extension To Higher-Order Modulations}

Although we focus primarily on BPSK signals so far, the aforementioned schemes can also achieve full diversity for higher-order modulations. For LAR and LAC, we observe that the power scaling coefficient $\alpha$ and combining weight $w$ we develop are independent of the detailed modulation schemes. Through some straightforward algebra, it is easy to show that our virtual channel model still fits for higher-order modulations, i.e., the quality of relay branch is approximately characterized by the worst channel inside. Therefore, full diversity can be achieved by following the same proof in the binary case.

As for the ON-OFF relaying, the decision rule (15) depends directly on the error probability at the relay node, which is hard to manipulate. Alternatively, we choose to extend the spirit of the threshold-based relaying (19) in a heuristic way. Recall that in the ON-OFF relaying scheme, the relay node is actually striving to prevent the error propagation by setting a stringent SNR threshold, such that the posterior error probability when the relay node passes the threshold test scales like $\operatorname{Pr}\left(\Phi_{\text {prop }} \mid \Phi_{\text {on }}\right)=\mathrm{O}\left(\Gamma^{-2}\right)$ as proved in (41). Intuitively, if the same scaling law is preserved for higherorder modulations, we can expect to achieve full diversity as well. As an example, we propose the following design for M-ary phase-shift keying (M-PSK) signals.

Proposition 5: For M-PSK signals, if we adopt the following decision rule for $\mathrm{ON}-\mathrm{OFF}$ relaying

$$
\Phi_{\text {on }}=\left\{\gamma_{s r, \text { min }} \geq g_{p s k}^{-1} \log \Gamma\right\}
$$

where $g_{p s k}=\sin ^{2}\left(\frac{\pi}{M}\right)$, then the posterior error probability $\operatorname{Pr}\left(\Phi_{\text {prop }} \mid \Phi_{\text {on }}\right)=\mathrm{O}\left(\Gamma^{-2}\right)$. 
Proof: The posterior PDF of $\gamma_{k r}$ given $\Phi_{o n}$ is

$$
\begin{aligned}
f\left(\gamma_{k r} \mid \Phi_{o n}\right) & =\frac{f\left(\gamma_{k r}\right)}{\operatorname{Pr}\left(\gamma_{k r} \geq g_{p s k}^{-1} \log \Gamma\right)} \\
& =\frac{1}{\lambda_{k r} \Gamma} \exp \left(-\frac{\gamma_{k r}-g_{p s k}^{-1} \log \Gamma}{\lambda_{k r} \Gamma}\right)
\end{aligned}
$$

for $\gamma_{k r} \geq g_{p s k}^{-1} \log \Gamma$ and $k=1,2$. The conditional symbol error probability for M-PSK signal is [18]

$$
\mathrm{P}_{e}(\gamma)=\frac{1}{\pi} \int_{0}^{\frac{M-1}{M} \pi} \exp \left(-\frac{g_{p s k} \gamma}{\sin ^{2} \theta}\right) d \theta
$$

Averaging the above probability over the posterior PDF of $\gamma_{k r}$ leads to (34) on the top of the previous page. Now we can conclude that $\operatorname{Pr}\left(\Phi_{\text {prop }} \mid \Phi_{\text {on }}\right) \leq \sum_{k \in\{1,2\}} \operatorname{Pr}\left(x_{r, k} \neq x_{k} \mid \Phi_{\text {on }}\right)=$ $\mathrm{O}\left(\Gamma^{-2}\right)$.

\section{Simulations}

In this section, we shall present some simulation results to validate our diversity analysis. Throughout simulations, we use the path loss model $\lambda=D^{-3}$, where $\lambda$ is the channel gain and $D$ is the distance between two terminals. Pair error probability is used as the performance metric, i.e., the probability that at least one of the source symbols is decoded incorrectly at the destination. We also simulate the genie-aided relaying and simple DF relaying as the references for comparisons. For genie-aided relaying, we suppose the relay node can perfectly detect the decoding error, and it only forwards the network-coded symbol using full power when the relay decoding is error-free, otherwise the relay node would stay idle. As for simple DF relaying, we assume the relay node just demodulates the received signal and then remodulates and forwards the detected symbol using full power no matter the decoding is reliable or not, and the combining weight is always set to 1 at the receiver side. Note that the diversity order of simple DF relaying is only 1 because $\operatorname{Pr}\left(\mathbf{x} \rightarrow\left(-x_{1}, x_{2}\right)\right) \geq \operatorname{Pr}\left(\mathbf{x} \rightarrow\left(-x_{1}, x_{2}\right), \Phi_{\text {prop }}, \Phi_{\text {on }}\right)=$ $\mathrm{O}\left(\Gamma^{-1}\right)$, where the inequality is due to (6) and the equality is obtained after plugging in (7) and (16b).

Fig. 3 shows the error performances in a symmetric network, where the distance between any two nodes is normalized. We observe that the local CSI based LAC only achieves a diversity order of 1 as direct transmission and simple DF, while all the other schemes achieve a diversity order of 2. The genie-aided relaying is the benchmark for all the practical schemes, thus having the best error performances. It is also observed that the performance of simplified ON-OFF relaying (19) is very close to that based on the exact decision rule (15) at all SNRs. The local CSI based LAR is slightly better than ON-OFF relaying through soft power scaling. The performances of the three global CSI based methods are very close. Comparatively, MLD is the best scheme among all, but it performs nearly the same as LAC which enjoys lower decoding complexity.

Then in Fig. 4 and Fig. 5 we present the error performances for two asymmetric networks. For the network with strong relay-destination channel and with strong source-relay channel, we set $D_{r d}=0.4$ and $D_{s r}=0.4$ respectively while

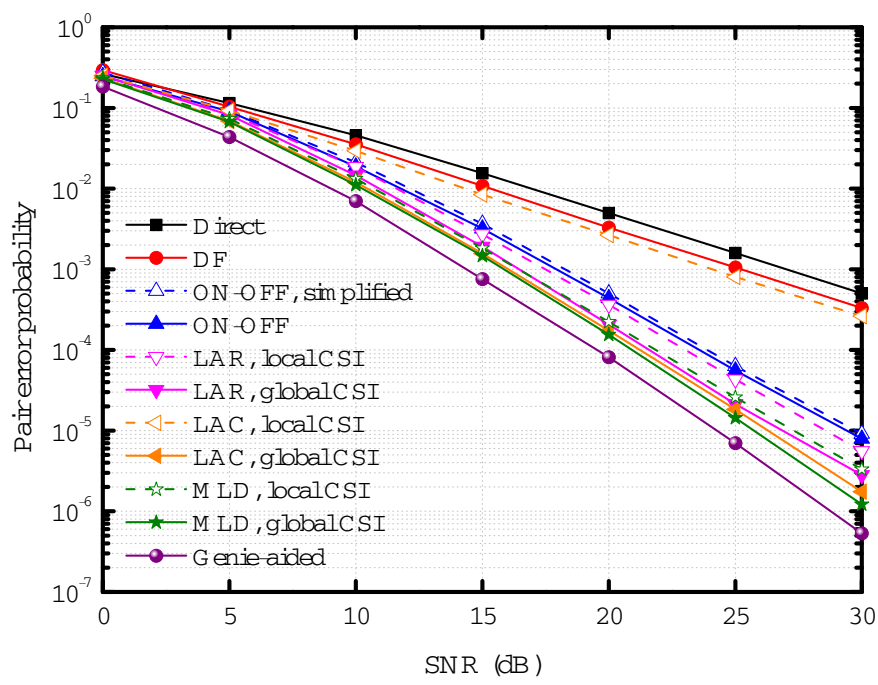

Fig. 3. Error performances of BPSK signal in a symmetric network.

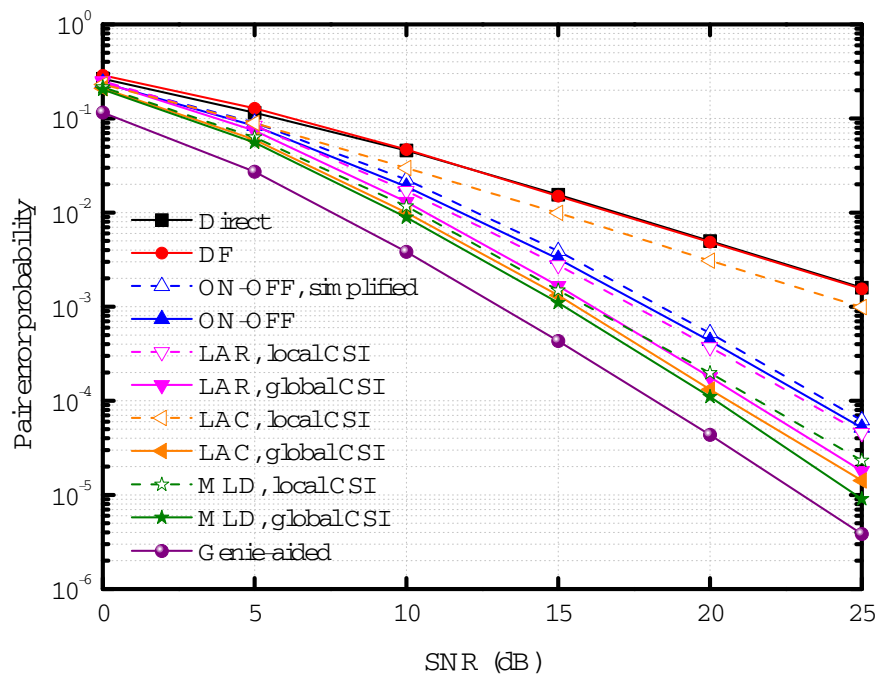

Fig. 4. Error performances of BPSK signal in an asymmetric network with strong relay-destination channel.

normalizing the other distances. For the former scenario, the source-relay channel is the system bottleneck. As the relay decoding is unreliable, the simple DF relaying performs almost the same as direct transmission. Besides, the performance gap between genie-aided relaying and all other schemes increases compared to the symmetric scenario, which reflects the importance of preventing error propagation. As for the network where the relay-destination channel is worse, the error propagation issue is comparatively mitigated. We observe in Fig. 5 that the simple DF and local CSI based LAC now have huge coding gain against direct transmission; however, the diversity order is still 1 . For all the remaining schemes, the performances are almost the same, and a diversity order of 2 is achieved.

Next we investigate the error performances with different relay positions. For the network topology, we place the destination at $(0,0)$, and locate the two source nodes at $\left(\frac{\sqrt{3}}{2}, \pm \frac{1}{2}\right)$, respectively. The relay node shall move along the $\mathrm{x}$-axis from $(0.2,0)$ to $(2,0)$. The error performance is shown in Fig. 6. It 


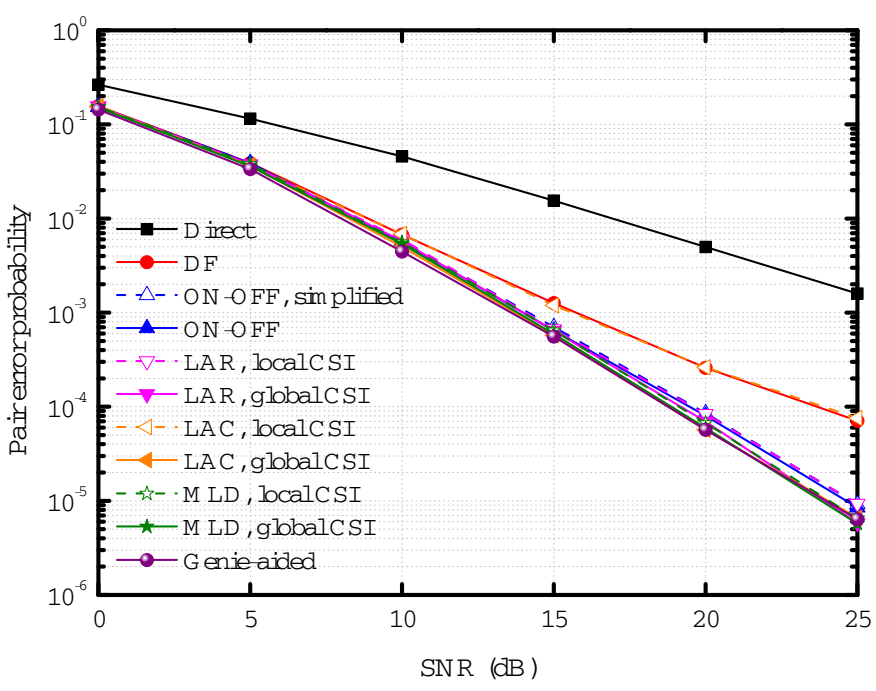

Fig. 5. Error performances of BPSK signal in an asymmetric network with strong source-relay channel.

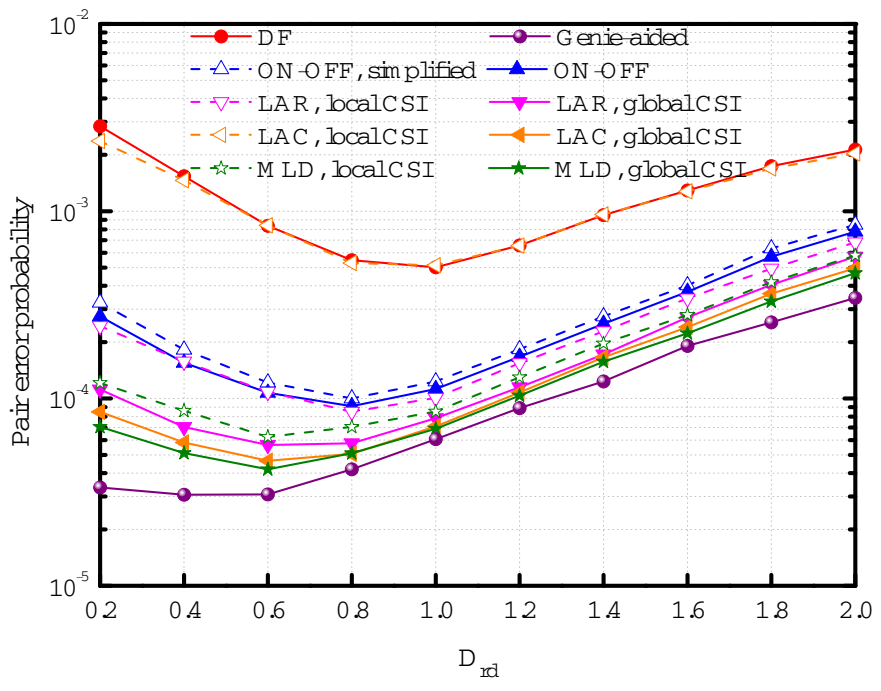

Fig. 6. Error performances of BPSK signal with $\Gamma=20 d B$ and different relay positions.

is observed that for all the schemes, the best performance is attained when the relay node is close to the source nodes, as the relay decoding error dominates the overall system performance. In all cases, the global CSI based schemes perform much better than their local CSI based counterparts, though at a price of higher signalling overhead.

For the same network, we also plot the relay power consumption ratio in Fig. 7. The simulation results are consistent with our analysis, i.e., the relay power consumption of ON-OFF relaying increases with SNR, while for LAR it is independent of SNR. For LAR, we observe that the power consumption is really low when the relay is close to the destination, since the source-relay link is comparatively unreliable; as the relay node moves far away from the destination, the relay node gradually increases its power until the relay-destination channel becomes the bottleneck. For $\mathrm{ON}-\mathrm{OFF}$ relaying and genie-aided relaying, the relay power consumption maximizes when the relay node is close to the

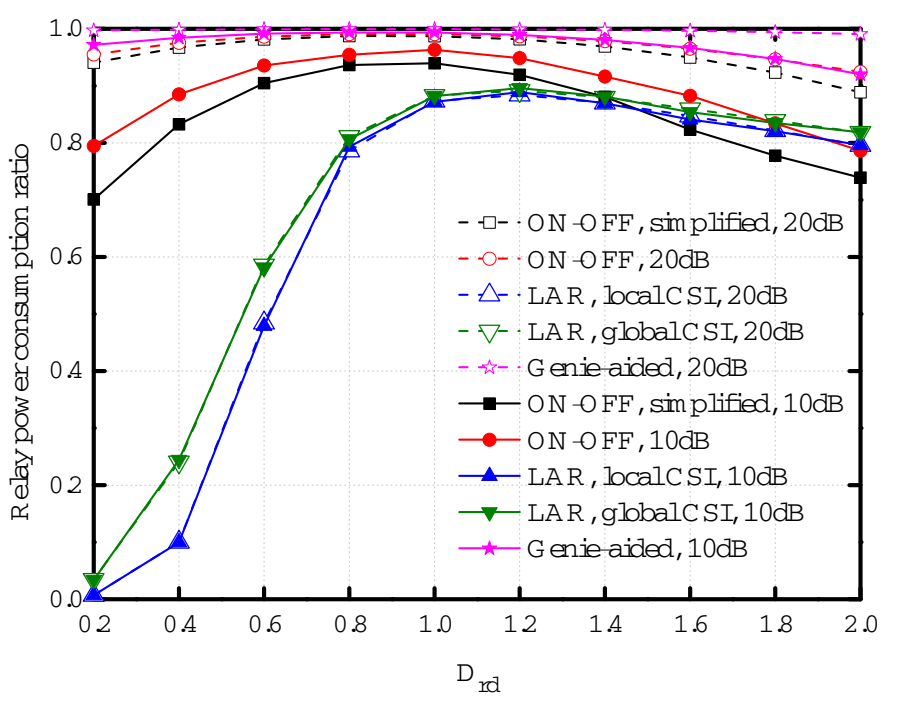

Fig. 7. Relay power consumption ratio with different relay positions.

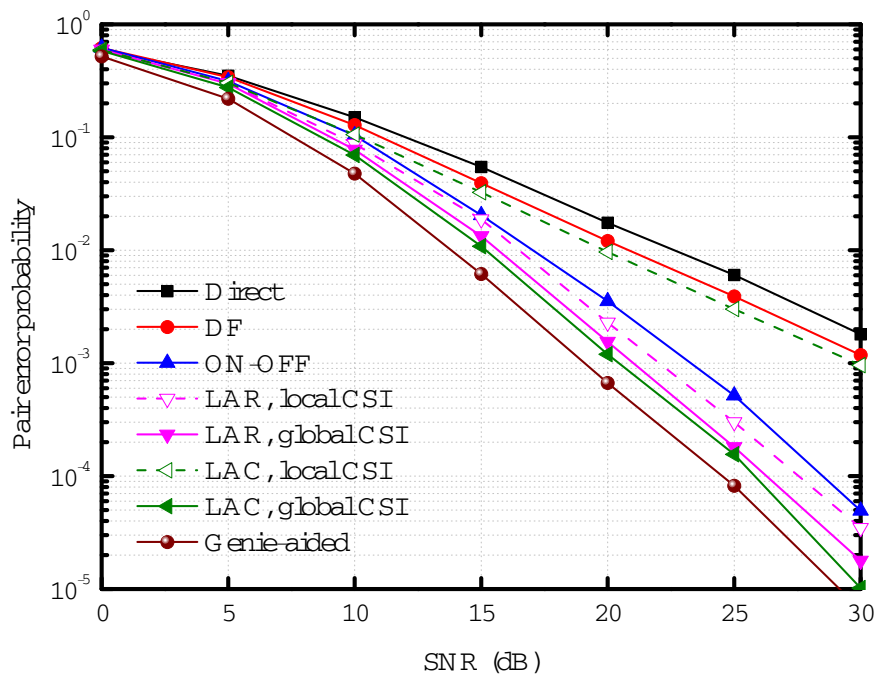

Fig. 8. Error performances of QPSK signal in a symmetric network.

source, in which case the relay decoding is really reliable and the chance of forwarding the message is high. We also observe that the soft power scaling based LAR is much more power efficient than the hard power scaling based ON-OFF relaying in most cases. This is because in the ON-OFF relaying, the relay node is always very conservative in forwarding the message so as to keep the posterior error probability low. Note that although the relay node always uses full power in the receiver-side schemes, better performances are also achieved compared to the relay-side schemes.

Finally, we study the error performances using higher-order modulations in Fig. 8 and Fig. 9. Clearly, a diversity order of 2 is achieved by all the schemes except local CSI based LAC, which validates our analysis.

\section{CONCLUSIONS AND FUtURE WORK}

In this work, we have proposed two power scaling schemes at the relay side and two detection schemes at the receiver side, respectively, that can mitigate error propagation and thus achieve full diversity for the wireless network-coded 


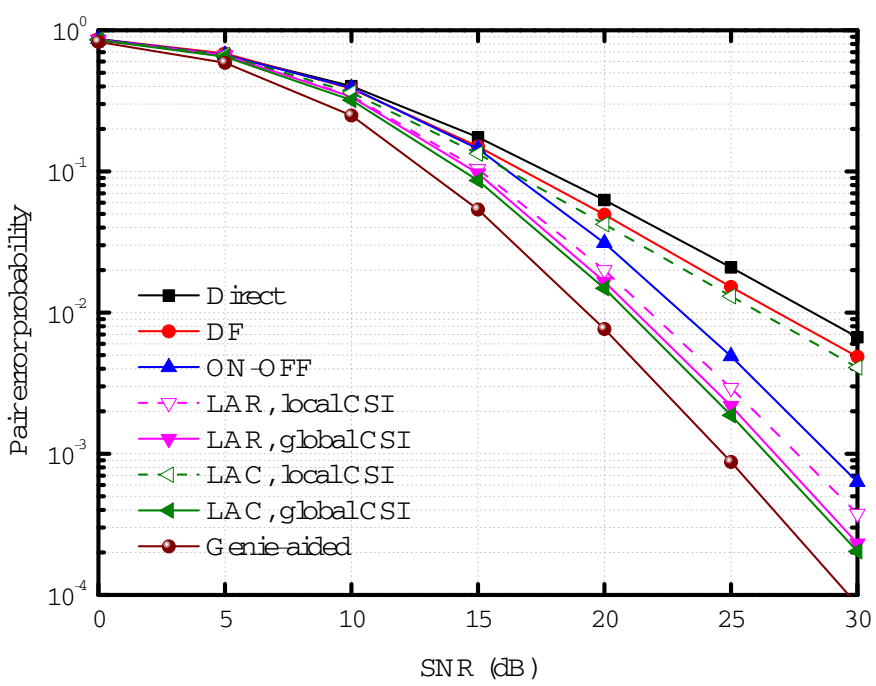

Fig. 9. Error performances of 8PSK signal in a symmetric network.

uplink. We showed that the receiver-side schemes generally has better error performances, whereas the relay-side schemes are more power efficient. We also demonstrated that there is a basic tradeoff between the error performance and signalling overhead to acquire channel conditions. We remark that the error propagation issue is addressed either at the relay side or at the receiver side in this work to achieve full diversity. One interesting issue for possible future consideration is how to jointly optimize the relaying scheme and detection scheme so as to improve the coding gain.

\section{APPENDIX}

\section{A. Proof of Proposition 1}

We prove the first part using our virtual channel model. As the relay branch is modeled as a point-to-point link with the channel input being $x_{\oplus}$, the whole transmitted codeword now becomes $\left(x_{1}, x_{2}, x_{\oplus}\right)$, where each symbol is delivered in different time slots. After some manipulations, it is easy to show that given any power scaling coefficient $\alpha$ employed at the relay side and any combining weight $w$ employed at the receiver side, the PEP can be approximated by (35) on the top of next page. For global CSI based LAR (13a), the virtual channel SNR is $\gamma_{s r d, v}=$ $\min \left(\gamma_{s r, \min }, \gamma_{r d}\right) \triangleq \gamma_{s r d, \min }$. Here $\gamma_{s r d, \min }$ follows exponential distribution with mean $\Gamma_{s r d, \min }=\lambda_{s r d, \min } \Gamma$, where $\lambda_{s r d, \text { min }}=\frac{\lambda_{1 r} \lambda_{2 r} \lambda_{r d}}{\lambda_{1 r} \lambda_{2 r}+\lambda_{1 r} \lambda_{r d}+\lambda_{2 r} \lambda_{r d}}$. After applying the Chernoff bound [19] on the Q-function and plugging in $w=1$, we can further obtain (36) on the top of next page, where $\Lambda_{i}$ and $r$ are the $i$ th non-zero eigenvalue and the rank of the diagonal matrix

$$
\left(\begin{array}{ccc}
\frac{\lambda_{1 d}\left|x_{1}-\hat{x}_{1}\right|^{2}}{4} & 0 & 0 \\
0 & \frac{\lambda_{2 d}\left|x_{2}-\hat{x}_{2}\right|^{2}}{4} & 0 \\
0 & 0 & \frac{\lambda_{s r d, \text { min }}\left|x_{\oplus}-\hat{x}_{\oplus}\right|^{2}}{4}
\end{array}\right),
$$

respectively. Because at least two diagonal elements are non-zero when an error event happens, we have $\max _{\hat{\mathbf{x}} \neq \mathbf{x}} \operatorname{Pr}(\mathbf{x} \rightarrow \hat{\mathbf{x}})=\mathrm{O}\left(\Gamma^{-2}\right)$, which completes the proof of the first part. For the second part, the PEP can be alternatively bounded using (6) after plugging in (8c), (8d), (11) and $\operatorname{Pr}\left(\Phi_{o f f}\right)=0$ as

$$
\begin{aligned}
& \operatorname{Pr}\left(\mathbf{x} \rightarrow\left(-x_{1}, x_{2}\right)\right) \\
& \left.\leq E\left[\left(Q \frac{\sqrt{2}\left(\gamma_{1 d}-\alpha \gamma_{r d}\right)}{\sqrt{\gamma_{1 d}+\alpha \gamma_{r d}}}\right) Q\left(\sqrt{2 \gamma_{s r, m i n}}\right)\right)\right] \\
& \quad+E\left[\left(Q\left(\sqrt{2\left(\gamma_{1 d}+\alpha \gamma_{r d}\right)}\right)\right)\right] .
\end{aligned}
$$

Note that the two source-relay channels have been put into one virtual channel with the virtual channel SNR being $\gamma_{s r, m i n}$, which still follows exponential distribution. As a result, we can follow the similar steps in [13] to show that both terms in (37) scale as $\mathrm{O}\left(\Gamma^{-2}\right)$ at high SNRs.

\section{B. Proof of Proposition 2}

Given the stated decision rule, we have

$$
\begin{aligned}
\operatorname{Pr}\left(\Phi_{\text {on }}\right) & =\prod_{k \in\{1,2\}} \operatorname{Pr}\left(\gamma_{k r} \geq \log \lambda_{T, k} \Gamma\right) \\
& =\exp \left(-\sum_{k \in\{1,2\}} \frac{\log \lambda_{T, k} \Gamma}{\lambda_{k r} \Gamma}\right) \\
& \stackrel{\log \lambda_{T, k} \Gamma}{\approx} \Gamma \stackrel{\sim}{\approx}^{\infty} 1,
\end{aligned}
$$

and $\operatorname{Pr}\left(\Phi_{\text {off }}\right)=1-\operatorname{Pr}\left(\Phi_{\text {on }}\right) \stackrel{\Gamma \rightarrow \infty}{\approx} \sum_{k \in 1,2} \frac{\log \lambda_{T, k} \Gamma}{\lambda_{k r} \Gamma}$. The posterior PDF of $\gamma_{k r}$ given $\Phi_{o n}$ is

$$
\begin{aligned}
f\left(\gamma_{k r} \mid \Phi_{o n}\right) & =\frac{f\left(\gamma_{k r}\right)}{\operatorname{Pr}\left(\gamma_{k r} \geq \log \lambda_{T, k} \Gamma\right)} \\
& =\frac{1}{\lambda_{k r} \Gamma} \exp \left(-\frac{\gamma_{k r}-\log \lambda_{T, k} \Gamma}{\lambda_{k r} \Gamma}\right)
\end{aligned}
$$

for $\gamma_{k r} \geq \log \lambda_{T, k} \Gamma$ and $k=1,2$. Now we can obtain

$\operatorname{Pr}\left(x_{r, k} \neq x_{k} \mid \Phi_{\text {on }}\right)$

$=\int_{\log \lambda_{T, k} \Gamma}^{\infty} Q\left(\sqrt{2 \gamma_{k r}}\right) \frac{1}{\lambda_{k r} \Gamma} \exp \left(-\frac{\gamma_{k r}-\log \lambda_{T, k} \Gamma}{\lambda_{k r} \Gamma}\right) d \gamma_{k r}$
$\leq \frac{1}{2 \lambda_{k r} \Gamma} \int_{\log \lambda_{T, k} \Gamma}^{\infty} \exp \left(-\gamma_{k r}\right) d \gamma_{k r}=\frac{1}{2 \lambda_{k r} \lambda_{T, k}} \Gamma^{-2}$, which leads to

$$
\begin{aligned}
\operatorname{Pr}\left(\Phi_{\text {prop }} \mid \Phi_{\text {on }}\right) & \leq \sum_{k \in\{1,2\}} \operatorname{Pr}\left(x_{r, k} \neq x_{k} \mid \Phi_{\text {on }}\right) \\
& \leq \frac{\lambda_{1 r} \lambda_{T, 1}+\lambda_{2 r} \lambda_{T, 2}}{2 \lambda_{1 r} \lambda_{2 r} \lambda_{T, 1} \lambda_{T, 2}} \Gamma^{-2}=\mathrm{O}\left(\Gamma^{-2}\right)(41)
\end{aligned}
$$

and $\operatorname{Pr}\left(\Phi_{\text {free }} \mid \Phi_{\text {on }}\right)=1-\operatorname{Pr}\left(\Phi_{\text {prop }} \mid \Phi_{\text {on }}\right) \stackrel{\Gamma{ }^{\infty}}{\approx} 1$. After plugging (17) and the above results back into (6), we have

$$
\begin{aligned}
\sum_{\hat{\mathbf{x}} \neq \mathbf{x}} & \operatorname{Pr}(\mathbf{x} \rightarrow \hat{\mathbf{x}}) \stackrel{\Gamma \rightarrow \infty}{\leq} \sum_{k \in\{1,2\}} \frac{\log \lambda_{T, k} \Gamma}{\lambda_{k r}} \frac{\lambda_{1 d}+\lambda_{2 d}}{4 \lambda_{1 d} \lambda_{2 d}} \Gamma^{-2} \\
& +\sum_{k \in\{1,2\}} \frac{\lambda_{r d}}{\lambda_{k d}+\lambda_{r d}} \frac{\lambda_{1 r} \lambda_{T, 1}+\lambda_{2 r} \lambda_{T, 2}}{2 \lambda_{1 r} \lambda_{2 r} \lambda_{T, 1} \lambda_{T, 2}} \Gamma^{-2} \\
& +\frac{\lambda_{1 d}+\lambda_{2 d}+\lambda_{r d}}{\lambda_{1 d} \lambda_{2 d} \lambda_{r d}} \frac{3}{16} \Gamma^{-2}
\end{aligned}
$$




$$
\operatorname{Pr}(\mathbf{x} \rightarrow \hat{\mathbf{x}}) \approx E\left[Q\left[\frac{\sum_{k \in\{1,2\}} \gamma_{k d}\left|x_{k}-\hat{x}_{k}\right|^{2}+w \alpha \gamma_{r d}\left|x_{\oplus}-\hat{x}_{\oplus}\right|^{2}}{\sqrt{2 \sum_{k \in\{1,2\}} \gamma_{k d}\left|x_{k}-\hat{x}_{k}\right|^{2}+2 \alpha^{2} w^{2} \gamma_{r d}^{2}\left|x_{\oplus}-\hat{x}_{\oplus}\right|^{2} / \gamma_{s r d, v}}}\right]\right.
$$

$$
\operatorname{Pr}(\mathbf{x} \rightarrow \hat{\mathbf{x}}) \leq E\left[\frac{1}{2} \exp \left(-\frac{\sum_{k \in\{1,2\}} \gamma_{k d}\left|x_{k}-\hat{x}_{k}\right|^{2}+\gamma_{s r d, m i n}\left|x_{\oplus}-\hat{x}_{\oplus}\right|^{2}}{4}\right)\right] \stackrel{\Gamma \rightarrow \infty}{\approx} \frac{1}{2}\left(\prod_{i=1}^{r} \Lambda_{i}\right)^{-1} \Gamma^{-r}
$$

$$
\operatorname{Pr}\left(\mathbf{x} \rightarrow\left(-x_{1}, x_{2}\right)\right)=\operatorname{Pr}\left(\frac{4 \operatorname{Re}\left(y_{1 d} \bar{h}_{1 d}^{*} x_{1}\right)}{N_{0}} \leq q\left(\operatorname{Pr}\left(\Phi_{\text {prop }}\right), \frac{4 \operatorname{Re}\left(y_{r d} \bar{h}_{r d}^{*} x_{1} x_{2}\right)}{N_{0}}\right)\right),
$$

Therefore, a diversity of 2 is achieved. To prove the second part, we need to find the optimum $\lambda_{T, k}$ for $k=1,2$ to minimize the above bound, i.e.,

$$
\lambda_{T, k}^{*}=\arg \min _{\lambda_{T, k}}\left(\log \lambda_{T, k} \frac{\lambda_{1 d}+\lambda_{2 d}}{2 \lambda_{1 d} \lambda_{2 d}}+\frac{1}{\lambda_{T, k}} \sum_{k \in\{1,2\}} \frac{\lambda_{r d}}{\lambda_{k d}+\lambda_{r d}}\right) .
$$

It is easy to check that $\lambda_{T, 1}^{*}=\lambda_{T, 2}^{*}=\lambda_{T}$ as given in (20).

\section{Proof of Proposition 4}

We only prove the case for local CSI based MLD, whose PEP strictly upper bounds that of global CSI based MLD. As $f\left(y_{r d} \mid x_{1}, x_{2}\right)=f\left(y_{r d} \mid-x_{1},-x_{2}\right)$, it is easy to show that $\operatorname{Pr}(\mathbf{x} \rightarrow-\mathbf{x})=\mathrm{O}\left(\Gamma^{-2}\right)$. Next we investigate the PEP $\operatorname{Pr}\left(\mathbf{x} \rightarrow\left(-x_{1}, x_{2}\right)\right)$. After some manipulations, we can obtain (44) on the top of this page, where

$$
q(\varepsilon, t)=\log \frac{\varepsilon+(1-\varepsilon) e^{t}}{\varepsilon e^{t}+(1-\varepsilon)} \approx\left\{\begin{array}{l}
\log \frac{1-\varepsilon}{\varepsilon}, t \geq \log \frac{1-\varepsilon}{\varepsilon} \\
t, \log \frac{\varepsilon}{1-\varepsilon} \leq t \leq \log \frac{1-\varepsilon}{\varepsilon} \\
\log \frac{\varepsilon}{1-\varepsilon}, t \leq \log \frac{\varepsilon}{1-\varepsilon}
\end{array} .\right.
$$

The last piece-wise linear approximation in (45) is proved in [5]. Define $Z=\frac{4 \operatorname{Re}\left(y_{1 d} \bar{h}_{1 d}^{*} x_{1}\right)}{N_{0}}$, which can be rewritten as the quadratic form of two independent complex Gaussian random variables. According to [20], the PDF of $Z$ is

$$
f(z)=\left\{\begin{array}{l}
\frac{a b}{a+b} e^{-a z}, z>0 \\
\frac{a b}{a+b} e^{b z}, \quad z \leq 0
\end{array}\right.
$$

where

$$
\left\{\begin{array}{l}
a=\sqrt{1+\Gamma_{1 d}^{-1}}-1 \stackrel{\Gamma \underset{\approx}{\approx}}{\approx} \frac{1}{2 \lambda_{1 d}} \Gamma^{-1} \\
b=\sqrt{1+\Gamma_{1 d}^{-1}}+1 \stackrel{\Gamma \rightarrow^{\infty}}{\approx} 2
\end{array} .\right.
$$

With the above PDF, it is easy to show that $\operatorname{Pr}(Z \leq-\log \Gamma) \stackrel{\Gamma \rightarrow \infty}{\approx} \frac{1}{4 \lambda_{1 d}} \Gamma^{-3}$ and $\operatorname{Pr}(Z \leq \log \Gamma) \quad=$

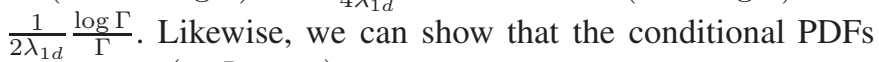
of $T=\frac{4 \operatorname{Re}\left(y_{r d} \bar{h}_{r d}^{*} x_{1} x_{2}\right)}{N_{0}}$ are

$$
f\left(t \mid \Phi_{\text {prop }}\right)=\left\{\begin{array}{l}
\frac{c d}{c+d} e^{-c t}, t>0 \\
\frac{c d}{c+d} e^{d t}, \quad t \leq 0
\end{array}\right.
$$

and

$$
f\left(t \mid \Phi_{\text {free }}\right)=\left\{\begin{array}{l}
\frac{c d}{c+d} e^{-d t}, \quad t>0 \\
\frac{c d}{c+d} e^{c t}, \quad t \leq 0
\end{array},\right.
$$

respectively, where

$$
\left\{\begin{array}{l}
c=\sqrt{1+\Gamma_{r d}^{-1}}-1 \stackrel{\Gamma \rightarrow \infty}{\underset{\approx}{*}} \frac{1}{2 \lambda_{r d}} \Gamma^{-1} \\
d=\sqrt{1+\Gamma_{r d}^{-1}}+1 \stackrel{\Gamma \rightarrow \infty}{\approx} 2
\end{array} .\right.
$$

Again it is easy to show that $\operatorname{Pr}\left(Z \leq T \mid \Phi_{\text {free }}\right) \stackrel{\Gamma \rightarrow \infty}{\approx} \frac{3}{16 \lambda_{1 d} \lambda_{r d}} \Gamma^{-2}=\mathrm{O}\left(\Gamma^{-2}\right)$. With the above results, we can obtain

$$
\begin{aligned}
& \operatorname{Pr}\left(\mathbf{x} \rightarrow\left(-x_{1}, x_{2}\right) \mid \Phi_{\text {prop }}\right) \\
& \quad=\operatorname{Pr}\left(Z \leq q\left(\operatorname{Pr}\left(\Phi_{\text {prop }}\right), T\right) \mid \Phi_{\text {prop }}\right) \\
& \quad \leq \operatorname{Pr}(Z \leq \nu) \stackrel{\Gamma \rightarrow \infty}{\approx} \operatorname{Pr}(Z \leq \log \Gamma)=\mathrm{O}\left(\frac{\log \Gamma}{\Gamma}\right)
\end{aligned}
$$

where

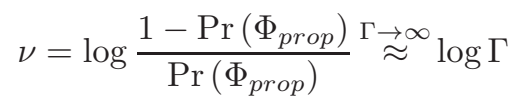

according to the high-SNR approximation of $\operatorname{Pr}\left(\Phi_{\text {prop }}\right)$ in (7). Besides, it is easy to show that

$$
\begin{aligned}
& \operatorname{Pr}\left(\mathbf{x} \rightarrow\left(-x_{1}, x_{2}\right) \mid \Phi_{\text {free }}\right) \\
& \approx \operatorname{Pr}\left(Z \leq \nu \leq T \mid \Phi_{\text {free }}\right)+\operatorname{Pr}\left(Z \leq-\nu, T \leq-\nu \mid \Phi_{\text {free }}\right) \\
& \quad+\operatorname{Pr}\left(Z \leq T,-\nu \leq T \leq \nu \mid \Phi_{\text {free }}\right) \\
& \quad \operatorname{Pr}\left(Z \leq T \mid \Phi_{\text {free }}\right)+\operatorname{Pr}(Z \leq-\nu)=\mathrm{O}\left(\Gamma^{-2}\right)
\end{aligned}
$$

where we use the piece-wise linear approximation (45). After plugging (7), (51) and (53) back into (6), we have $\operatorname{Pr}\left(\mathbf{x} \rightarrow\left(-x_{1}, x_{2}\right)\right)=\mathrm{O}\left(\frac{\log \Gamma}{\Gamma^{2}}\right)$. Consequently, a diversity order of 2 is achieved.

\section{REFERENCES}

[1] K. J. R. Liu, A. K. Sadek, W. Su, and A. Kwasinski, Cooperative Communications and Networking. Cambridge University Press, 2008.

[2] J. N. Laneman, D. N. C. Tse, and G. W. Wornell, "Cooperative diversity in wireless networks: efficient protocols and outage behavior," IEEE Trans. Inf. Theory, vol. 50, no. 12, pp. 3062-3080, Dec. 2004.

[3] R. Ahlswede, N. Cai, S.-Y. R. Li, and R. W. Yeung, "Network information flow," IEEE Trans. Inf. Theory, vol. 46, no. 4, pp. 1204-1216, July 2000.

[4] H. Q. Lai and K. J. R. Liu, "Space-time network coding," IEEE Trans. Signal Process., vol. 59, no. 4, pp. 1706-1718, Apr. 2011.

[5] D. Q. Chen and J. N. Laneman, "Modulation and demodulation for cooperative diversity in wireless systems," IEEE Trans. Wireless Commun., vol. 5, no. 7, pp. 1785-1794, July 2006.

[6] W. Guan and K. J. R. Liu, "Performance analysis of two-way relaying with non-coherent differential modulation," IEEE Trans. Wireless Commun., vol. 10, no. 6, pp. 2004-2014, June 2011. 
[7] Y. Chen, S. Kishore, and J. Li, "Wireless diversity through network coding," in Proc. 2006 IEEE Wireless Commun. Netw. Conf., vol. 3, pp. 1681-1686.

[8] Z. Han, X. Zhang, and H. V. Poor, "Cooperative transmission protocols with high spectral efficiency and high diversity order using multiuser detection and network coding," IEEE Trans. Wireless Commun., vol. 8, no. 5, pp. 2352-2361, May 2009.

[9] M. Xiao and M. Skoglund, "Multiple-user cooperative communications based on linear network coding," IEEE Trans. Commun., vol. 58, no. 12, pp. 3345-3351, Dec. 2010.

[10] F. A. Onat, A. Adinoyi, Y. Fan, H. Yanikomeroglu, J. Thompson, and I. Marsland, "Threshold selection for SNR-based selective digital relaying in cooperative wireless networks," IEEE Trans. Wireless Commun., vol. 7, no. 11, pp. 4226-4237, Nov. 2008.

[11] T. Himsoon, W. P. Siriwongpairat, W. F. Su, and K. J. R. Liu, "Differential modulation with threshold-based decision combining for cooperative communications," IEEE Trans. Signal Process., vol. 55, no. 7, pp. 3905-3923, July 2007.

[12] S. L. H. Nguyen, A. Ghrayeb, G. Al-Habian, and M. Hasna, "Mitigating error propagation in two-way relay channels with network coding," IEEE Trans. Wireless Commun., vol. 9, no. 11, pp. 3380-3390, Nov. 2010.

[13] T. Wang, G. B. Giannakis, and R. Q. Wang, "Smart regenerative relays for link-adaptive cooperative communications," IEEE Trans. Commun., vol. 56, no. 11, pp. 1950-1960, Nov. 2008.

[14] T. Wang, A. Cano, G. B. Giannakis, and J. N. Laneman, "Highperformance cooperative demodulation with decode-and-forward relays," IEEE Trans. Commun., vol. 55, no. 7, pp. 1427-1438, July 2007.

[15] A. Nasri, R. Schober, and M. Uysal, "Error rate performance of networkcoded cooperative diversity systems," in Proc. 2010 IEEE Global Commun. Conf., pp. 1-6.

[16] M. D. Selvaraj, R. K. Mallik, and R, Goel, "Optimum receiver performance with binary phase-shift keying for decode-and-forward relaying," IEEE Trans. Veh. Technol., vol. 60, no. 4, pp. 1948-1953, May 2011.

[17] M. Iezzi, M. D. Renzo, and F. Graziosi, "Closed-form error probability of network-coded cooperative wireless networks with channel-aware detectors," 2011 IEEE Global Commun. Conf.

[18] M. K. Simon and M. S. Alouini, "A unified approach to the performance analysis of digital communications over generalized fading channels," Proc. IEEE, vol. 86, no. 9, pp. 1860-1877, Sep. 1998.

[19] J. Proakis, Digital Communications, 4th edition. McGraw-Hill, 2001.
[20] K. H. Biyari and W. C. Lindsey, "Statistical distributions of hermitian quadratic forms in complex Gaussian variables," IEEE Trans. Inf. Theory, vol. 39, no. 3, pp. 1076-1082, May 1993.

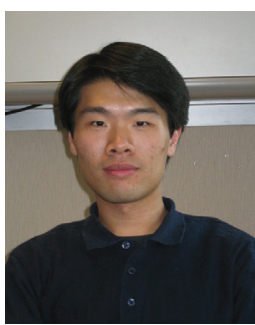

Wei Guan (S'12) received the B.S. in Electrical Engineering and Finance (double degree) in 2006, and M.S. (with highest honor) in Electrical Engineering in 2009, both from Shanghai JiaoTong University, Shanghai, China. Now he is a Ph.D. student in the Department of Electrical and Computer Engineering at University of Maryland, College Park. His current research interests are in the areas of wireless communications and networks, including cooperative communications and network coding. He received the 1st Prize in the 18th National Physics Contest, Shanghai, and the A. James Clark School of Engineering Distinguished Graduate Fellowship from University of Maryland, College Park in 2009.

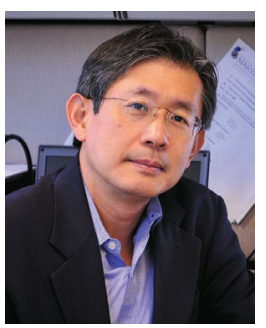

K. J. Ray Liu (F'03) was named a Distinguished Scholar-Teacher of University of Maryland, College Park, in 2007, where he is Christine Kim Eminent Professor of Information Technology. He leads the Maryland Signals and Information Group conducting research encompassing broad areas of signal processing and communications with recent focus on cooperative communications, cognitive networking, social learning and networks, and information forensics and security.

Dr. Liu is the recipient of numerous honors and awards including IEEE Signal Processing Society Technical Achievement Award and Distinguished Lecturer. He also received various teaching and research recognitions from University of Maryland including university-level Invention of the Year Award; and Poole and Kent Senior Faculty Teaching Award and Outstanding Faculty Research Award, both from A. James Clark School of Engineering. An ISI Highly Cited Author in Computer Science, Dr. Liu is a Fellow of IEEE and AAAS.

Dr. Liu is President of IEEE Signal Processing Society where he has served as Vice President - Publications and Board of Governor. He was the Editor-inChief of IEEE Signal Processing Magazine and the founding Editor-in-Chief of EURASIP Journal on Advances in Signal Processing. 